\title{
Arbuscular mycorrhizal fungi alter above- and below-ground chemical defense expression differentially among Asclepias species
}

\author{
Rachel L. Vannette ${ }^{1,2 *}$, Mark D. Hunter ${ }^{2}$ and Sergio Rasmann ${ }^{3+}$ \\ 1 Biology Department, Stanford University, Stanford, CA, USA \\ 2 Department of Ecology and Evolutionary Biology, University of Michigan, Ann Arbor, MI, USA \\ ${ }^{3}$ Department of Ecology and Evolutionary Biology, University of Lausanne, Lausanne, Switzerland
}

\section{Edited by:}

Maria J. Pozo, Consejo Superior de Investigaciones Cientificas, Spain

Reviewed by:

Nicole M. Van Dam, Radboud University Nijmegen, Netherlands

Eduardo De La Pena, Ghent

University, Belgium

*Correspondence:

Rachel L. Vannette, Biology Department, Stanford University, 371 Serra Mall, Stanford, CA 94305, USA

e-mail: raleva@stanford.edu

${ }^{\dagger}$ Present address:

Sergio Rasmann, Ecology and

Evolutionary Biology, University of

California, Irvine, USA
Below-ground (BG) symbionts of plants can have substantial influence on plant growth and nutrition. Recent work demonstrates that mycorrhizal fungi can affect plant resistance to herbivory and the performance of above- (AG) and BG herbivores. Although these examples emerge from diverse systems, it is unclear if plant species that express similar defensive traits respond similarly to fungal colonization, but comparative work may inform this question. To examine the effects of arbuscular mycorrhizal fungi (AMF) on the expression of chemical resistance, we inoculated 8 species of Asclepias (milkweed)-which all produce toxic cardenolides-with a community of AMF. We quantified plant biomass, foliar and root cardenolide concentration and composition, and assessed evidence for a growth-defense tradeoff in the presence and absence of AMF. As expected, total foliar and root cardenolide concentration varied among milkweed species. Importantly, the effect of mycorrhizal fungi on total foliar cardenolide concentration also varied among milkweed species, with foliar cardenolides increasing or decreasing, depending on the plant species. We detected a phylogenetic signal to this variation; AMF fungi reduced foliar cardenolide concentrations to a greater extent in the clade including A. curassavica than in the clade including A. syriaca. Moreover, AMF inoculation shifted the composition of cardenolides in AG and BG plant tissues in a species-specific fashion. Mycorrhizal inoculation changed the relative distribution of cardenolides between root and shoot tissue in a species-specific fashion, but did not affect cardenolide diversity or polarity. Finally, a tradeoff between plant growth and defense in non-mycorrhizal plants was mitigated completely by AMF inoculation. Overall, we conclude that the effects of AMF inoculation on the expression of chemical resistance can vary among congeneric plant species, and ameliorate tradeoffs between growth and defense.

Keywords: plant-herbivore interactions, mycorrhizal fungi, plant defense, above-below-ground interactions, growth-defense tradeoff, root defense, phylogenetic signal

\section{INTRODUCTION}

Plants are preyed upon by a diverse community of enemies, including pathogens and herbivores, which damage plant tissue both above- (AG) and below-ground (BG) and can severely reduce plant fitness. In response, plants have evolved a diversity of defenses, including the ability to tolerate or resist such attack (van der Meijden et al., 1988; Karban and Baldwin, 1997). We focus here on plant resistance to herbivory, which includes the expression of toxic secondary compounds, those that reduce nutrient assimilation by herbivores, or even attract predators of such herbivores. Although specialist herbivores exhibit adaptations to ameliorate the effects of toxins produced by their host plants, these same toxins can also negatively affect herbivore growth. For example, plants in the genus Asclepias produce potent cardenolides, and although specialist herbivores, including Danaus plexippus and Tetraopes tetraophthalmus, can tolerate and sequester these compounds (Agrawal et al., 2012), high concentrations or novel combinations of cardenolides can reduce performance on milkweed hosts (Zalucki et al., 2001b; Rasmann and Agrawal, 2011a). As a result, variation in the expression of defense compounds by plants, whether driven by genotype or phenotypic plasticity, can influence the performance of both specialist and generalist herbivores.

Classical theories of plant defense expression AG describe how both the expression and evolution of defense compounds depend on the abiotic environment and levels of herbivory (Bryant et al., 1983; Coley et al., 1985; Herms and Mattson, 1992). However, recent evidence indicates that the presence and abundance of microorganisms may also affect the expression of plant defenses AG and BG (Rieske, 2001; Thamer et al., 2011). Microbial symbionts in roots, for instance, may affect plant resistance systemically through a number of hormonal, nutritive, or signaling pathways (Vannette and Hunter, 2009; Pineda et al., 2010). Although plants in natural settings are nearly always 
associated with such microorganisms (Smith and Read, 2008; Partida-Martinez and Heil, 2011), the consequences of plantmicrobe interactions are rarely incorporated into plant defense theories (but see Bennett et al., 2006; Vannette and Hunter, 2011). As a result, understanding microbial effects on plant resistance and plant-herbivore interactions should have broad implications for our understanding of both the evolution and expression of plant defense.

We focus here on plant associations with Glomeromycetes, also known as arbuscular mycorrhizal fungi (AMF). These ubiquitous symbionts colonize the roots of the majority of land plant species examined for the association (Smith and Read, 2008), often increasing plant phosphorus and nitrogen uptake in exchange for carbon transferred to the fungus. This exchange leads directly to predictions for the expression of nutrient-limited defenses (Vannette and Hunter, 2011) and implies that AMF may modify the shape of the theorized growth-defense tradeoff (De Deyn et al., 2009; Vannette and Hunter, 2011). A growing number of studies have examined the influence of AMF on plant defense expression and herbivory, and a few trends have emerged. For example, inoculation with AMF often increases the performance of specialist chewing insects, and decreases the performance of generalist insects (Gehring and Whitham, 1994; Koricheva et al., 2009). In one of the few studies to link the expression of resistance traits to herbivore performance, suppression of AMF in the roots of Plantago lanceolata decreased the concentration of two major iridoid constituents in leaves (Gange and West, 1994). Reductions in chemical defense in P. lanceolata were associated with significant increases in consumption by the generalist folivore Arctia caja. Despite the potential importance of plantassociated microbes for plant-herbivore interactions, their effects on plant defense are hardly generalizable. For example, AMF species differentially affect expression of iridoid glycosides in P. lanceolata (Bennett et al., 2009) and of cardenolides in Asclepias syriaca (Vannette and Hunter, 2011). However, when AMF identity is kept constant, does plant resistance among species respond similarly to AMF inoculation? One study reported that inoculation with Glomus intraradices increased the growth of the insect herbivore Spodoptera littoralis to an equivalent degree among 6 unrelated grassland plants (Kempel et al., 2010), although the mechanism of effect was not investigated. However, a field study demonstrated high levels of variation among prairie plant species in tolerance to herbivory in the presence of AMF (Kula et al., 2005). Similarly, AMF inoculation differentially affected several genotypes of Datura stamonium in their level of tolerance to herbivory (Garrido et al., 2010), and full-sibling families of Plantago lanceolata also responded differently in the expression of iridoid glycosides with AMF inoculation (De Deyn et al., 2009). However, relatively few studies actually examine the effects of AMF on the expression of resistance among congeners. As a result, predicting the outcome of multitrophic interactions based on plant identity remains a challenge.

In order to incorporate the effects of AMF on plant defense into ecological and evolutionary theory, we should examine whether inoculation with mycorrhizal fungi affects similarly plants that share defense compounds. Previous work using A. syriaca demonstrates that an increase in AMF abundance is often associated with increased plant phosphorus status and with expression of phosphorus-limited defenses, including cardenolides and trichomes (Tao and Hunter, 2012; Vannette and Hunter, 2013). Will such patterns hold among other species of Asclepias? Plant species vary in the degree to which their phenotypes respond to AMF (Klironomos, 2003; Janos, 2007) and this may include their chemical resistance traits.

In addition, given the importance of root-feeding herbivores in both natural and managed ecosystems (Hunter, 2008), increasing efforts to study root defense have demonstrated that putative plant resistance traits in roots significantly influence BG herbivore performance (Rasmann and Agrawal, 2011a). AMF colonization may also further increase plant protection against BG feeders (Gange and West, 1994; Vannette and Rasmann, 2012). However, the expression of secondary compounds BG could also negatively affect mycorrhizal associations (Strauss and Irwin, 2004). As a result, balancing the potential benefits of root defense and mycorrhizal gains may pose a tradeoff for many plants. Additionally, initial results suggest that mycorrhizal fungi may differentially affect AG and BG defense (De Deyn et al., 2009), but the effects of AMF on the relative expression of resistance compounds between AG and BG tissues is unclear. Such effects could be ecologically relevant, with different consequences for the performance of shoot and root feeders.

To examine the effects of AMF on plant resistance both AG and BG, we assessed how mycorrhizal inoculation affects the expression of cardenolides, a class of steroid glycosides, among eight Asclepias species. Here, we used a comparative phylogenetic approach to disentangle factors that generate variation in AMF-mediated multitrophic interactions. Phylogenetic methods provide powerful tools with which to compare species-specific responses within single experiments. In this case, while controlling for relatedness, we can consider whether plant responses to AMF are predictable within a single clade. Specifically, we asked the following questions: first, does inoculation with mycorrhizal fungi differentially influence the expression of defense compounds among congeneric plant species? Second, does association with AMF influence the distribution of defense compounds among plant roots and shoots? Third, are the effects of AMF on plant biomass and defense similar in AG and BG tissues? Finally, do AMF affect the relationship between plant growth and the expression of secondary chemistry?

We used milkweeds, in the genus Asclepias, to examine the effects of AMF inoculation on the expression of plant resistance compounds in roots and foliar tissue. Plant species in the genus Asclepias are an ideal system with which to address this question, as nearly all species produce cardenolides, toxic molecules which can disrupt the sodium and potassium flux in animal cells when ingested (Malcolm, 1991). These compounds occur in all milkweed tissues, including roots (Rasmann et al., 2009), and have selected for a community of specialist herbivores shared among Asclepias species. Despite insect behavioral and physiological adaptations to reduce cardenolide exposure and toxicity (Dussourd and Eisner, 1987; Holzinger and Wink, 1996), empirical evidence suggests that cardenolides continue to be detrimental to both AG (Zalucki et al., 2001a; Agrawal, 2005; de Roode et al., 2011) and BG herbivores (Rasmann et al., 2011). Asclepias species 
are also colonized by mycorrhizal fungi, which can influence growth and the expression of resistance phenotypes, including cardenolide expression (Vannette and Hunter, 2011; Vannette and Rasmann, 2012).

\section{MATERIALS AND METHODS EXPERIMENTAL DESIGN}

In order to assess the influence of AMF on AG and BG expression of defense among Asclepias species, we selected eight species all producing variable levels of cardenolides (Rasmann and Agrawal, 2011b), and grew them in the presence and absence of mycorrhizal fungi. Because of high phylogenetic signal for cardenolide production across the American milkweeds (Agrawal and Fishbein, 2008), the species were also chosen in order to cover most of the phylogenetic variation. Additionally, all eight species are known to be associated with root herbivores, the longhorn beetle larvae in the genus Tetraopes (Farrell and Mitter, 1998), live in very distinct habitats (Rasmann and Agrawal, 2011a), and associate with mycorrhizal fungi (Vannette and Rasmann, 2012).

Plants were grown as described previously (Vannette and Rasmann, 2012). Briefly, seeds were cold stratified and germinated in petri dishes. Individual seedlings $(n=10-26$ replicates per plant species depending on germination success) were transplanted into $10 \mathrm{~cm}$ diameter plastic pots in a mixture of low nutrient, autoclaved potting soil (Metro-Mix 360, Metro-Mix Sun Gro Horticulture Canada CM Ltd., Vancouver, BC, Canada) and perlite (3:1 parts potting soil: perlite), and grown in a single growth chamber ( $10 \mathrm{~h}$ daylight, $26^{\circ} \mathrm{C}$ day: $17^{\circ} \mathrm{C}$ night). The two mycorrhizal treatments were prepared by combining the soil mixture with live or autoclaved mycorrhizal inoculum consisting of a combination of root fragments and spores. We obtained inoculum of cosmopolitan fungal species known to associate with Asclepias syriaca (Vannette and Hunter, 2011) including Rhizophagus intraradices, Funneliformis mosseae, G. aggregatum, and Claroideoglomus etunicatum (Stockinger et al., 2009) from Mycorrhizal Applications (Grants Pass, OR, USA) and Claroideoglomus etunicatum cultured on the roots of Sorghum plants. Inocula from both sources were thoroughly mixed and made up 1/6 of the total pot volume, between layers of sterile soil. Although these species were not isolated from the rhizosphere of Asclepias, they have been found in forests, grassland, wetlands, and arable fields (Öpik et al., 2006), habitats in which all Asclepias species used here are also found. Seedlings were planted with either mycorrhizal fungi or autoclaved inoculum, watered ad libitum, and grown in a growth chamber for 12 weeks.

\section{HARVEST AND CARDENOLIDE ANALYSIS}

Plants were harvested after 12 weeks of growth. Nearly all plants received damage by sciarid fly larvae present in the growth chamber (Vannette and Rasmann, 2012). To quantify the number of larvae in roots, we placed potato discs near plant roots for 3 days and counted fly larvae as they colonized the discs. Following larval counts, plant tissue was harvested, separated into AG and BG tissues, dried at $40^{\circ} \mathrm{C}$ and weighed. We verified mycorrhizal colonization by staining roots and examining them for mycorrhizal structures [data presented in Vannette and Rasmann (2012)].
To quantify the expression of cardenolides in AG and BG tissue, we used previously described methods to analyze cardenolide concentrations in foliar and root tissue (Zehnder and Hunter, 2007). Fine root and foliar tissues for each plant were ground separately, extracted in methanol for $1 \mathrm{~h}$, and the subsequent extract dried and re-suspended in methanol with digitoxin as an internal standard. Each sample was filtered through a $0.2 \mu \mathrm{m}$ filter and cardenolide compounds separated using UPLC (Waters Inc.) on an Acquity BEH C18 column $(1.7 \mu \mathrm{m}, 2.1 \times 50 \mathrm{~mm}$, Waters, Milford, MA, USA). Each $2 \mu$ l injection was eluted at a constant flow of $0.7 \mathrm{ml} / \mathrm{min}$ with a gradient of acetronitrile $(\mathrm{ACN})$ and water, maintained at $20 \% \mathrm{ACN}$ for $3 \mathrm{~min}$, increasing to $45 \%$ ACN through the 9 min run. Peaks were detected by a diode array detector at $218 \mathrm{~nm}$, and absorbance spectra were recorded from 200 to $400 \mathrm{~nm}$. Peaks with symmetrical absorbance between 218 and $222 \mathrm{~nm}$ were quantified as cardenolides (Malcolm and Zalucki, 1996). Cardenolide concentrations were calculated using the digitoxin internal standard and initial sample mass, and total cardenolides were calculated as the sum of individual peaks.

\section{CALCULATIONS AND STATISTICAL ANALYSES}

We assessed whether inoculation with mycorrhizal fungi influenced several indices of cardenolide expression. For both AG and BG tissues, we calculated cardenolide diversity and average polarity (Lefevre et al., 2010; Rasmann and Agrawal, 2011b). We used Shannon's index to calculate cardenolide diversity. We calculated cardenolide non-polarity following Sternberg et al. (2012); evidence suggests that non-polar cardenolides and a high diversity of cardenolides produce higher toxicity to a variety of organisms than do polar cardenolides or low diversity mixes (Fordyce and Malcolm, 2000; Zehnder and Hunter, 2007; Sternberg et al., 2012). Non-polarity was calculated for each sample by summing the relative peak areas multiplied by each peaks' retention time. Response variables were assessed for normality and homogeneity of variance and log-transformed if needed to reduce heteroscedasticity. Using Two-Way ANOVA, we examined if Asclepias species, AMF treatment, or their interaction affected each response variable, including total cardenolide concentration, diversity, and polarity. To control for the potential effect of root-feeding fly larvae on plant defense expression, we included larval density (the number of larvae divided by the total root biomass per plant) in the model as a covariate; it was never a significant variable, and was dropped from all subsequent analyses. Based on preliminary results (Figure 1), we explored whether the effects of AMF on cardenolide expression AG varied between phylogenetic clades. We used post-hoc contrasts comparing the effects of AMF on the five species in the A. curassavica clade to the effects of AMF on the clade containing A. syriaca. Contrasts were coded and tested using the multcomp package in $\mathrm{R}$.

To assess if AMF influenced the composition (i.e., identity and relative abundance) of cardenolide compounds AG or BG, we used non-metric multidimensional scaling (NMDS) implemented in the vegan package (Oksanen et al., 2012) in $\mathrm{R} \mathrm{v}$ 2.15.2 (Team, 2012). Differences in cardenolide composition among species, AMF treatment, and their interaction were tested using a permutational ANOVA (PERMANOVA), using the adonis function in the vegan package (Oksanen et al., 2012). The 


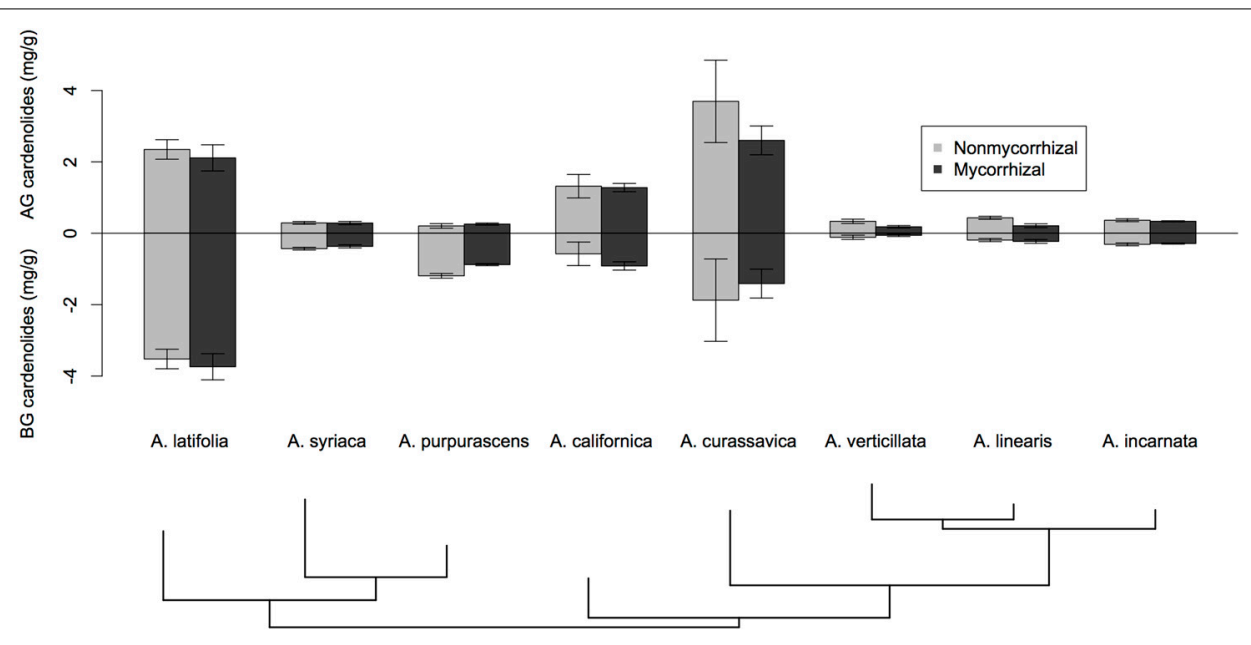

FIGURE 1 | The effect of AMF inoculation on cardenolide concentrations above- and below-ground among eight species of Asclepias. Bars are mean \pm 1SE. Bottom of figure shows the phylogenetic relatedness of Asclepias species used in this study. Phylogram pruned after Fishbein et al. (2011).

Bray-Curtis metric was used to calculate dissimilarity among samples for both the NMDS and PERMANOVA, although results were robust to other distance metrics. We also assessed the relationship between cardenolide expression in root and foliar tissue by regressing species' means for total AG and BG cardenolides using a Phylogenetic Least Squares (PGLS) framework. We estimated phylogenetic signal in average AG and BG growth and defense traits by calculating lambda values (Table S1), and phylogenetic regressions were estimated using the pgls function implemented in caper (Orme et al., 2012).

To explore if AMF influenced the distribution of cardenolides between plant roots and shoots, we calculated the root:shoot ratio of total cardenolide concentrations and used two-way ANOVA to examine the effects of Asclepias species, AMF treatment, and their interaction on root: shoot ratio of plant biomass and cardenolide concentrations. We also examined if AMF affected (a) plant biomass and (b) cardenolide concentration similarly AG and BG. For both mycorrhizal and non-mycorrhizal plants, we calculated mean values for AG and BG biomass and total cardenolide concentration for each species, then took the difference between mycorrhizal and non-mycorrhizal values. The difference in AG biomass was regressed against the difference in BG biomass (and similarly AG against BG cardenolides) using the PGLS framework. In this way, we could distinguish the effect of AMF inoculation on trait expression from variation arising from relatedness among species. Finally, to examine if AMF affected hypothesized growth-defense tradeoffs among plant individuals, we regressed plant biomass against whole-plant cardenolide concentration, and examined if AMF inoculation influenced the strength or direction of this relationship.

\section{RESULTS}

Asclepias species varied substantially in their expression of cardenolides in both foliar tissue [Figure 1; Species $F_{(7,117)}=21.26$, $p<0.001$ ] and in fine roots [Figure 1; Species $F_{(7,113)}=21.08$, $p<0.0001]$. Mycorrhizal inoculation affected the expression of cardenolides AG in a species-specific fashion [Figure 1;
Species $\left.\times \operatorname{AMF} F_{(7,117)}=2.25, p=0.034\right]$. Post-hoc contrasts indicated that AMF inoculation decreased cardenolide concentration AG to a greater extent in the A. curassavica clade (5 species) than in the clade containing A. syriaca [3 species; $t=2.62, p=0.009$; Figure 1]. In contrast, AMF tended to increase root cardenolide concentration across all species to a small extent, but this effect did not vary significantly among Asclepias species [Figure 1; AMF treatment $F_{(1,149)}=5.46, p=$ 0.02 ; Species $\left.\times \operatorname{AMF} F_{(7,149)}=1.05, p=0.39\right]$. Asclepias species varied in the diversity of cardenolides expressed, both AG and BG [Figure 2A; AG Species $F_{(7,114)}=0.41, p<0.001$; BG Species $\left.F_{(7,128)}=17.21, p<0.001\right]$. Similarly, Asclepias species varied in cardenolide polarity both AG and BG [Figure 2B; AG Species $F_{(7,117)}=10.44, p<0.001$; BG Species $F_{(7,128)}=76.71, p<$ $0.001]$. Notably, the cardenolides expressed by all Asclepias species are much more non-polar AG than they are BG (Figure 2B). Mycorrhizal inoculation did not affect cardenolide diversity either AG or BG [Figure 2A; AG AMF $F_{(1,114)}=0.08, p=0.77$; BG AMF $\left.F_{(1,128)}=0.26, p=0.61\right]$. Mycorrhizal inoculation increased cardenolide non-polarity in AG plant tissue, although this change was driven largely by changes in A. californica and A. purpurascens [Figure 2B; $\operatorname{AMF} F_{(1,114)}=4.29, p=0.04$ ], but inoculation did not significantly affect the polarity of cardenolides in root tissue [Figure 2B; $\operatorname{AMF} F_{(1,121)}=0.90, p=0.34$ ].

Cardenolide composition (the identity and relative abundance of molecular types) differed dramatically between roots and shoots, so were analyzed separately (Table S2). NMDS illustrated that Asclepias species varied in the composition of cardenolides expressed AG, and that AMF colonization shifted foliar cardenolide composition in a species-specific fashion [Figure 3A; PERMANOVA Species $F_{(7,114)}=18.08, P<0.001$; $\operatorname{AMF} F_{(1,114)}=2.46, P=0.021$; Species $\times \operatorname{AMF} F_{(7,114)}=1.65$, $P=0.007]$. Similarly, Asclepias species varied in cardenolide composition BG, and AMF also shifted cardenolide composition in a species-specific fashion [Figure 3B, PERMANOVA Species $F_{(7,121)}=18.3, \quad P<0.001 ;$ AMF $F_{(1,121)}=1.55, \quad P=0.08$; Species $\left.\times \operatorname{AMF} F_{(7,121)}=1.24, P=0.04\right]$. 

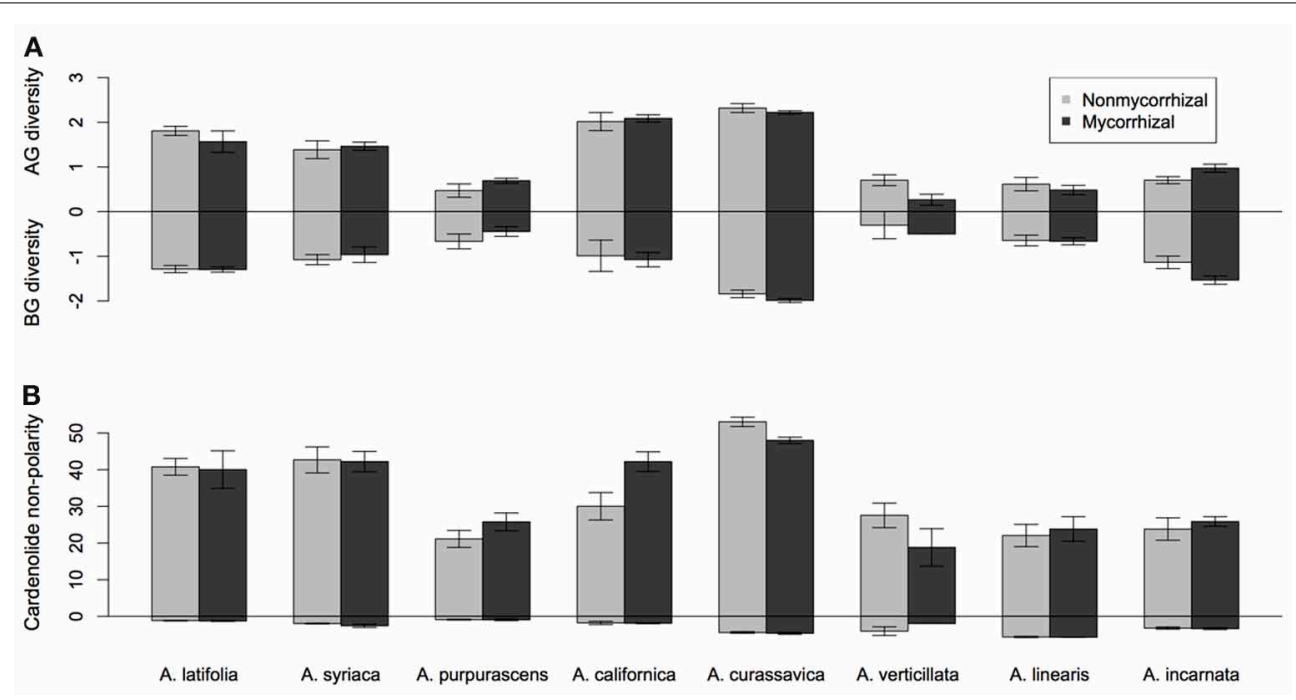

FIGURE 2 | Effect of AMF inoculation on above-ground (AG) and below-ground (BG) metrics of (A) cardenolide diversity (H), and (B) non-polarity, a measure of cardenolide toxicity (see text for details). Bars display the mean $\pm 1 \mathrm{SE}$ for each species.
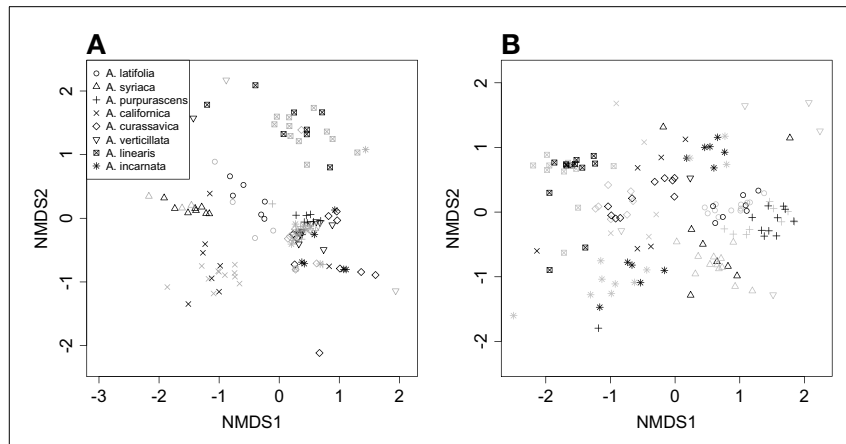

FIGURE 3 | NMDS plot illustrating variation in the composition of (A) above-ground foliar and (B) below-ground root cardenolides among eight milkweed species (Asclepias spp.), and the effects of AMF on cardenolide composition. Points represent plant individuals, where black points are mycorrhizal plants and gray points are non-mycorrhizal points.

Pagel's lambda indicated that most species traits exhibit some level of phylogenetic signal (Lambda close to 1), with the exception of AG biomass and BG cardenolide diversity (Table S1). However, 95\% confidence intervals usually included both zero and one, likely due to the small number of species used to estimate lambda values.

Among Asclepias species, the expression of cardenolides AG and BG was positively correlated (Figure 4), even when phylogeny was taken into account [PGLS regression $F_{(2,6)}=7.8$, $p=0.02]$. This relationship did not differ between mycorrhizal and non-mycorrhizal plants (Figure 4). We also examined the effects of mycorrhizal inoculation on root:shoot biomass ratio. While root:shoot allocation varied among species $\left[F_{(7,112)}=11.63, p<0.001\right]$, the effect of AMF inoculation on root:shoot ratio varied in direction and magnitude among species $\left[\right.$ Figure 5A; Species $\left.\times \operatorname{AMF} F_{(7,112)}=5.94, p<0.001\right]$. Asclepias

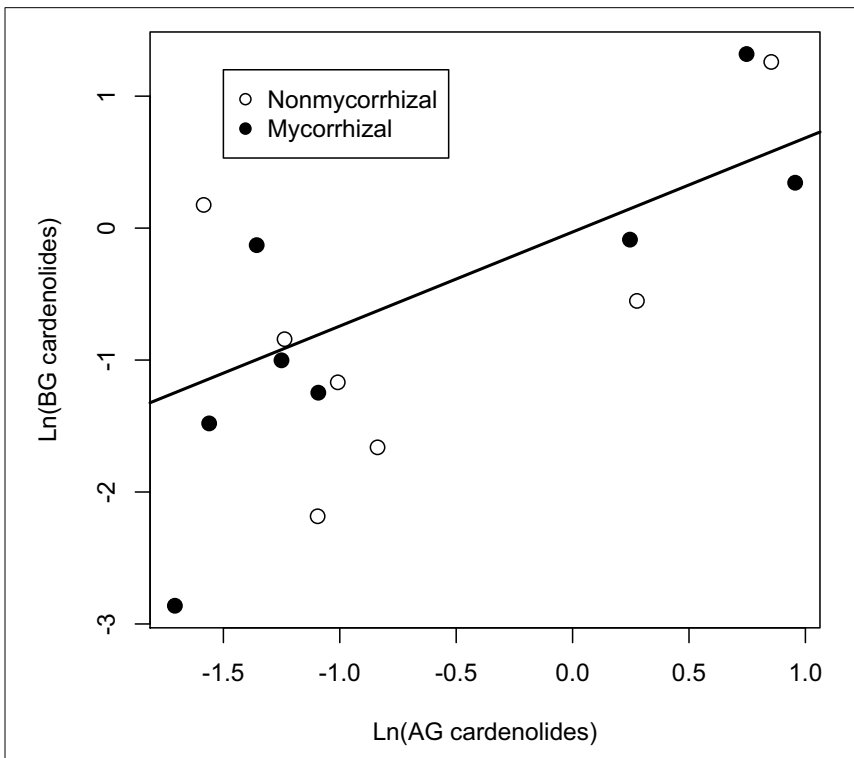

FIGURE 4 | Regressions between above (AG) and below-ground (BG) cardenolides among $\mathbf{8}$ species of Asclepias. Points represent the mean level of cardenolides in plants that were inoculated with AMF or sterilized inoculum (non-mycorrhizal). Line represents PGLS best-fit line.

species varied in the ratio of root:foliar cardenolides [Species $\left.F_{(7,110)}=4.95, p<0.001\right]$, and mycorrhizal inoculation also influenced the ratio of total cardenolides in roots to those in plant leaves in a species-specific fashion [Figure 5B, Species $\times$ AMF $\left.F_{(7,110)}=5.44, p=0.008\right]$.

In addition, we used the PGLS framework to explore the relationship between AMF effects on biomass and cardenolides in foliar and root tissue (Table S1). The effect of AMF inoculation on shoot biomass was strongly positively correlated with the effect on root biomass among plant species, even when phylogenetic 


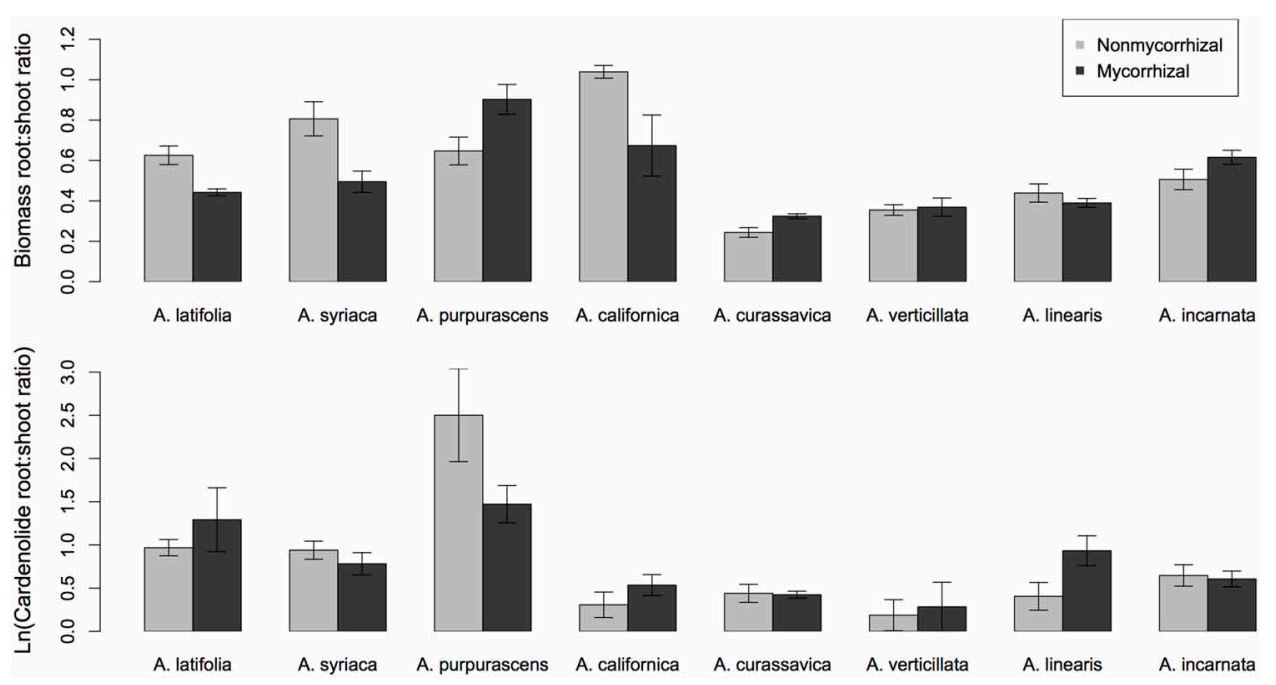

FIGURE 5 | Effects of mycorrhizal inoculation on (A) root:shoot biomass ratio, and (B) ratio of total cardenolide concentrations in roots:foliar tissue, among eight species of Asclepias. Cardenolide ratios were log-transformed after calculation.
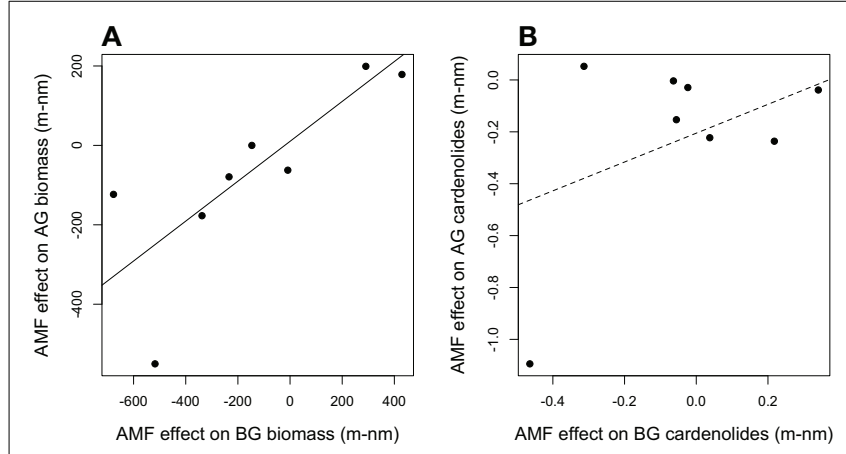

FIGURE 6 | The relationship between the effects of AMF on Asclepias (A) above-ground (AG) and below-ground (BG) biomass and (B) total cardenolide concentration. Effects are calculated as mycorrhizal ( $\mathrm{m}$ ) minus non-mycorrhizal $(\mathrm{nm})$ values. Each point represents the average effect of AMF for one of eight species of Asclepias. PGLS regression lines are shown, and the solid line indicates that the regression is significant at $p<0.05$

relatedness was accounted for [Figure 6A; PGLS $F_{(2,6)}=12.8$, $\left.R^{2}=0.62, p=0.01\right]$. However, the effect of AMF on foliar cardenolide concentration was unrelated to its effect on roots [Figure 6B; $F_{(2,6)}=1.5, R^{2}=0.06, p=0.26$ ].

Finally, we identified a growth-defense tradeoff among non-mycorrhizal plants. Specifically, total plant biomass and whole-plant cardenolide concentration were negatively correlated (Figure 7A), explaining $11 \%$ of the variation in whole-plant cardenolide concentration $\left[F_{(1,60)}=8.62, R^{2}=0.11, p=0.004\right]$. However, when we assessed the same relationship in mycorrhizal plants, no significant relationship was detected $\left[F_{(1,62)}=0.41\right.$, $R^{2}=0, p=0.52$ ] (Figure 7B). A similar trend was detected at the whole-plant level, but was not statistically significant (data not shown).
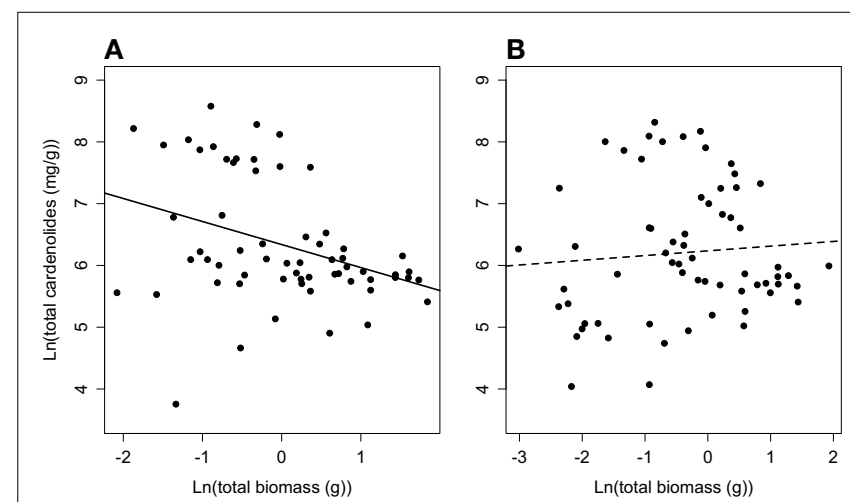

FIGURE 7 | Phenotypic correlation between total plant biomass and whole-plant cardenolide concentration for (A) non-mycorrhizal and (B) mycorrhizal Asclepias plants.

\section{DISCUSSION}

Our results demonstrate that mycorrhizal fungi differentially affect the expression and composition of AG and BG plant chemical defense traits, and that in large part, the effects of AMF inoculation are species-specific across the eight species of Asclepias examined here. In addition, we found evidence that AMF inoculation may alleviate the phenotypic tradeoff between plant growth and defense. Taken together, these results confirm that AMF can affect the traits that mediate interactions between plants and herbivores. However, variation in the magnitude and direction of responses to AMF within this single genus of plants was notable. Examining which effects of AMF are consistent among species and which vary may improve our ability to generalize about the multitrophic effects of AMF across species.

Surprisingly, the plants whose foliar cardenolide concentrations were affected most by AMF (e.g., A linearis, A. verticillata) showed little response in their root cardenolide concentrations 
to AMF colonization. Other species (e.g., A. purpurascens and A. incarnata) showed the reverse trend (Figures 1A,B). This suggests that not only do plant species vary in phenotypic response to inoculation with AMF (Klironomos, 2003), but plant species also vary in the tissues that are most responsive to inoculation with AMF. Variation among plant species in their responses to AMF can result from differences between coevolved and novel combinations of mutualists (Klironomos, 2003; Johnson et al., 2010) and shared evolutionary history among plants (Reinhart et al., 2012). Phylogenetic relatedness could explain some variation seen in our experiment: estimates of lambda were close to one for most cardenolide traits (Table S1), similar to previously documented patterns (Agrawal and Fishbein, 2008). Furthermore, the effect of AMF on AG cardenolide expression differed among clades within the Asclepias genus. However, the limited number of plant species included in this study hampered our ability to detect a significant phylogenetic signal in most plant traits. We suggest two non-mutually exclusive explanations for the variation among species in their phenotypic responses to AMF that we observed in our current study. First, Asclepias species may differentially associate with a subset of the AMF species available, as has been demonstrated with two forb species growing in grassland systems (Vandenkoornhuyse et al., 2002), and AMF species or communities may exert differential effects on plant phenotype (Bennett and Bever, 2007; Vannette and Hunter, 2011). Indeed, using the same plant species and inoculum, we found strong variation among Asclepias species in the number of arbuscules colonizing the roots, independent of phylogeny (Vannette and Rasmann, 2012), which may indicate differential association between Asclepias species and AMF. Second, plant species may vary in their phenotypic response to inoculation with the same fungal species because of differential resource transfer (Johnson et al., 2010), variation in hormonal or signaling response to colonization, or variation in plant ability to limit carbon flow to fungi (Grman, 2012). However, the variation in phenotypic response among Asclepias species indicates that, despite strong effects of mycorrhizal fungi on chemical defense in some species, predicting AMF-induced changes in plant resistance traits across taxa may prove difficult. An examination of the nutrient benefits transferred among partners, or other plant physiological traits may prove helpful in forming predictions for future work.

Given the demonstrated importance of AMF to the induced defense responses of plants (Kempel et al., 2010; Barber, 2012), it is likely that AMF-mediated changes in induction may also be important in resistance to herbivory among Asclepias species. Future work should examine if mycorrhizal fungi affect induction in a similar way among Asclepias species, and if AMF-plant relationships interact with latitudinal patterns in the evolution of induction (Rasmann and Agrawal, 2011b).

In addition to species-level variation in response to AMF, we observed differential effects of AMF on the expression of chemical compounds BG and AG. The effects of AMF on AG cardenolide concentration and composition varied among Asclepias species, but AMF inoculation had statistically similar and weak effects among species on BG cardenolide concentration and composition. However, AMF effects on cardenolide composition were species-specific both AG and BG (Figure 3). In addition, although the effects of AMF on AG and BG biomass were strongly positively correlated, AMF effects on the total concentration of cardenolides AG and BG were unrelated (Figure 6). This suggests that different mechanisms control plant biomass and secondary metabolite allocation when associating with AMF in Asclepias. Additionally, cardenolide profiles differed dramatically between foliar and root tissues, confirming previous results in milkweed (Nelson et al., 1981; Rasmann and Agrawal, 2011b; Agrawal et al., 2012) and broad patterns of defense across plant species overall (van Dam, 2009). Although cardenolides can be synthesized in shoot tissue (Groeneveld et al., 1990), the major site of synthesis is not well-understood, and it is possible that cardenolide biosynthesis may be ultimately fine-tuned in different plant organs (Manson et al., 2012). Despite the notable speciesspecific variation that we recorded, the differential effects of AMF on AG and BG resistance expression suggest a mechanism by which root and shoot feeders respond differently to mycorrhizal inoculation, as has been shown previously (Gange, 2001). Although we have already demonstrated that AMF convey protection against BG feeders (Vannette and Rasmann, 2012), future work should examine differences in effects of AMF between AG and BG herbivores. Investigating the role of AMF on insects that display differential mother-offspring feeding strategies, as seen in milkweed specialist Tetraopes beetles, may be an informative avenue to explore differential effects on AG and BG root feeders.

Cardenolides expressed in fine roots were considerably less non-polar than were cardenolides expressed in foliage (Figure 2B), despite the similar concentration and diversity of cardenolides in AG and BG tissues (Figures 1A,B, 2A). Nonpolar cardenolides are generally considered more toxic than are polar cardenolides, because non-polar forms may pass more easily across cell membranes (Fordyce and Malcolm, 2000). So our results suggest that cardenolides in fine roots occur at equal concentrations, but in less toxic forms, than those in leaves. This finding contrasts with results described by Rasmann and Agrawal (2011b), who found comparable cardenolide polarity in AG and BG tissues. However, only fine roots (the site of AMF colonization) were used in the current analysis, whereas the previous study also included rhizome, which is stem tissue. Work from multiple systems has demonstrated that main and storage roots are often well-protected compared to fine roots (van Dam, 2009), which may suggest differential pressure by herbivores or differences in root development, longevity, or value to plants. In addition, this pattern may also indicate plant adaptation to association with fungal mutualists, which occurs in fine roots, but not rhizome. Microbial mutualists in fine roots may be susceptible to non-polar compounds, compete for carbon otherwise used for defense synthesis, or even receive such compounds for their own protection in the presence of fungivores (Duhamel et al., 2013).

Finally, mycorrhizal inoculation altered the putative growthdefense tradeoff that we documented in non-mycorrhizal plants. In previous work, we hypothesized that mycorrhizal fungi may modify growth-defense tradeoffs by either increasing nutrient acquisition by plants or increasing $\mathrm{C}$ cost at high 
levels of AMF abundance (Vannette and Hunter, 2011). Although we did not measure plant nutrient status in this experiment, our results lend tentative support to this hypothesis. Indeed, the general difficulty in identifying growthdefense tradeoffs in natural populations may be due to plant associations with herbivores or mutualists, including mycorrhizal fungi that modify predicted growth-defense relationships (Herms and Mattson, 1992).

\section{CONCLUSIONS}

The results described here demonstrate that despite pervasive effects of AMF on plant resistance expression, significant variation exists among congeners in their responses to mycorrhizal colonization. As a result, we suggest that exploring the ecological or molecular correlates of such variation may lead to a better understanding of how BG microorganisms influence multitrophic interactions.

\section{REFERENCES}

Agrawal, A. A. (2005). Natural selection on common milkweed (Asclepias syriaca) by a community of specialized insect herbivores. Evol. Ecol. Res. 7, 651-667.

Agrawal, A. A., and Fishbein, M. (2008). Phylogenetic escalation and decline of plant defense strategies. Proc. Natl. Acad. Sci. U.S.A. 105, 10057-10060. doi: 10.1073/pnas.0802368105

Agrawal, A. A., Petschenka, G., Bingham, R. A., Weber, M. G., and Rasmann, S. (2012). Toxic cardenolides: chemical ecology and coevolution of specialized plant-herbivore interactions. New Phytol. 194, 28-45. doi: 10.1111/ j.1469-8137.2011.04049.x

Barber, N. A. (2012). Arbuscular mycorrhizal fungi are necessary for the induced response to herbivores by Cucumis sativus. J. Plant Ecol. 93, 1560-1570. doi: 10.1093/jpe/rts026

Bennett, A. E., Alers-Garcia, J., and Bever, J. D. (2006). Three-way interactions among mutualistic mycorrhizal fungi, plants, and plant enemies: hypotheses and synthesis. Am. Nat. 167, 141-152. doi: $10.1086 / 499379$

Bennett, A. E., and Bever, J. D. (2007). Mycorrhizal species differentially alter plant growth and response to herbivory. Ecology 88, 210-218. doi: $\quad 10.1890 / 0012-9658(2007)$ 88[210:MSDAPG]2.0.CO;2

Bennett, A. E., Bever, J. D., and Bowers, M. D. (2009). Arbuscular mycorrhizal fungal species suppress inducible plant responses and alter defensive strategies following herbivory. Oecologia 160, 771-779. doi: 10.1007/s00442-009-1338-5

Bryant, J. P., Chapin, F. S., and Klein, D. R. (1983). Carbon nutrient balance of boreal plants in relation to vertebrate herbivory. Oikos 40, 357-368. doi: $10.2307 / 3544308$

Coley, P. D., Bryant, J. P., and Chapin, F. S. (1985). Resource availability and plant antiherbivore defense. Science 230, 895-899. doi: 10.1126/science.230.4728.895

De Deyn, G. B., Biere, A., van der Putten, W. H., Wagenaar, R., and Klironomos, J. N. (2009). Chemical defense, mycorrhizal colonization and growth responses in Plantago lanceolata L. Oecologia 160, 433-442. doi: 10.1007/s00442-009$1312-2$

de Roode, J. C., Rarick, R. M., Mongue, A. J., Gerardo, N. M., and Hunter, M. D. (2011). Aphids indirectly increase virulence and transmission potential of a monarch butterfly parasite by reducing defensive chemistry of a shared food plant. Ecol. Lett. 14, 453-461. doi: 10.1111/j.1461-0248.2011.01604.x

Duhamel, M., Pel, R., Ooms, A., Bücking, H., Jansa, J., Ellers, J., et al. (2013). Do fungivores trigger the transfer of protective metabolites from host plants to arbuscular mycorrhizal hyphae? Ecology. doi: 10.1890/12-1943.1

Dussourd, D. E., and Eisner, T. (1987). Vein-cutting behavior - insect counterplay to the latex defense of plants. Science 237, 898-901. doi: 10.1126/science. 3616620

Farrell, B. D., and Mitter, C. (1998). The timing of insect/plant diversification: might Tetraopes (Coleoptera: Cerambycidae) and Asclepias (Asclepiadaceae) have co-evolved? Biol. J. Linn. Soc. 63, 553-577.

Fishbein, M., Chuba, D., Ellison, C., Mason-Gamer, R. J., and Lynch, S. P. (2011). Phylogenetic relationships of Asclepias (Apocynaceae) inferred

\section{ACKNOWLEDGMENTS}

We thank Daisy Johnson for help with data collection; M. Fishbein for sharing phylogenetic information about the milkweeds; Anurag Agrawal for providing Asclepias seeds and logistic support during plant growth. We thank two anonymous reviewers for helpful comments. Our research was supported by a postdoctoral fellowship from the Swiss National Science Foundation (PA0033-121483 to Sergio Rasmann), support from the University of Michigan Department of Ecology and Evolutionary Biology and a National Science Foundation DDIG to Rachel L. Vannette and NSF Grants DEB-0814340 and DEB-1256115 to Mark D. Hunter.

\section{SUPPLEMENTARY MATERIAL}

The Supplementary Material for this article can be found online at: http://www.frontiersin.org/Plant-Microbe_ Interaction/10.3389/fpls.2013.00361/abstract

from non-coding chloroplast DNA sequences. Syst. Bot. 36, 1008-1023. doi: 10.1600/036364411X605010

Fordyce, J. A., and Malcolm, S. B. (2000). Specialist weevil, Rhyssomatus lineaticollis, does not spatially avoid cardenolide defenses of common milkweed by ovipositing into pith tissue. J. Chem. Ecol. 26, 2857-2874.

Gange, A. C. (2001). Species-specific responses of a root- and shootfeeding insect to arbuscular mycorrhizal colonization of its host plant. New Phytol. 150, 611-618. doi: 10.1046/j.1469-8137.2001.00137.x

Gange, A. C., and West, H. M. (1994). Interactions between arbuscular mycorrhizal fungi and foliar-feeding insects in Plantago lanceolata, L. New Phytol. 128, 79-87. doi: 10.1111/j.14698137.1994.tb03989.x

Garrido, E., Bennett, A. E., Fornoni, J., and Strauss, S. Y. (2010). Variation in arbuscular mycorrhizal fungi colonization modifies the expression of tolerance to aboveground defoliation. J. Ecol. 98, 43-49. doi: 10.1111/j.1365-2745. 2009.01586.x

Gehring, C. A., and Whitham, T. G. (1994). Interactions between aboveground herbivores and the mycorrhizal mutualists of plants. Trends Ecol. Evol. 9, 251-255. doi: 10.1016/0169-5347(94)90290-9

Grman, E. (2012). Plant species differ in their ability to reduce allocation to non-beneficial arbuscular mycorrhizal fungi. Ecology 93, 711-718. doi: 10.1890/11-1358.1

Groeneveld, H. W., van den Berg, B., Elings, J. C., and Seykens, D. (1990). Cardenolide biosynthesis from malonate in Asclepias curassavica. Phytochemistry 29, 3479-3486. doi: $\quad 10.1016 / 0031-9422(90) 85$ 261-D

Herms, D. A., and Mattson, W. J. (1992). The dilemma of plants - to grow or defend. Q. Rev. Biol. 67, 283-335. doi: 10.1086/417659

Holzinger, F., and Wink, M. (1996). Mediation of cardiac glycoside insensitivity in the Monarch butterfly (Danaus plexippus): role of an amino acid substitution in the ouabain binding site of $\mathrm{Na}+, \mathrm{K}+$-ATPase. J. Chem. Ecol. 22, 1921-1937. doi: 10.1007/ BF02028512

Hunter, M. D. (2008). "Root herbivory in forest ecosystems," in Root Feeders, an Ecosystem Perspective, eds S. N. Johnson and P. J. Murray (Ascot: CAB Biosciences), 68-95.

Janos, D. P. (2007). Plant responsiveness to mycorrhizas differs from dependence upon mycorrhizas. Mycorrhiza 17, 75-91. doi: 10.1007/s00572-006-0094-1

Johnson, N. C., Wilson, G. W. T., Bowker, M. A., Wilson, J. A., and Miller, R. M. (2010). Resource limitation is a driver of local adaptation in mycorrhizal symbioses. Proc. Natl. Acad. Sci. U.S.A. 107, 2093-2098. doi: 10.1073/pnas.0906710107

Karban, R., and Baldwin, I. T. (1997). Induced Responses to Herbivory, 1st Edn. Chicago, IL: University of Chicago Press. doi: 10.7208/ chicago/9780226424972.001.0001

Kempel, A., Schmidt, A. K., Brandl, R., and Schadler, M. (2010). Support from the underground: induced plant resistance depends on arbuscular mycorrhizal fungi. Funct. Ecol. 24, 293-300. doi: 10.1111/j.13652435.2009.01647.x

Klironomos, J. N. (2003). Variation in plant response to native and 
exotic arbuscular mycorrhizal fungi. Ecology 84, 2292-2301. doi: 10.1890/02-0413

Koricheva, J., Gange, A. C., and Jones, T. (2009). Effects of mycorrhizal fungi on insect herbivores: a metaanalysis. Ecology 90, 2088-2097. doi: 10.1890/08-1555.1

Kula, A. A. R., Hartnett, D. C., and Wilson, G. W. T. (2005). Effects of mycorrhizal symbiosis on tallgrass prairie plant-herbivore interactions. Ecol. Lett. 8, 61-69. doi: 10.1111/j.1461-0248.2004.00690.x

Lefevre, T., Oliver, L., Hunter, M. D., and de Roode, J. C. (2010). Evidence for transgenerational medication in nature. Ecol. Lett. 13, 1485-1493. doi: 10.1111/j.1461-0248.2010.01537.x

Malcolm, S. B., (1991). "Cardenolidemediated interactions between plants and herbivores," in Herbivores: Their Interactions with Secondary Metabolites, eds G. A. Rosenthal and M. R. Berenbaum (San Diego, CA: Academic Press), 251-296. doi: 10.1016/B978-0-12597183-6.50012-7

Malcolm, S. B., and Zalucki, M. P. (1996). Milkweed latex and cardenolide induction may resolve the lethal plant defence paradox. Entomol. Exp. Appl. 80, 193-196. doi: $\quad 10.1111 / j .1570-7458.1996$. tb00916.x

Manson, J. S., Rasmann, S., Halitschke, R., Thomson, J. D., and Agrawal, A. A. (2012). Cardenolides in nectar may be more than a consequence of allocation to other plant parts: a phylogenetic study of Asclepias. Funct. Ecol. 26, 1100-1110. doi: 10.1111/j.1365-2435.2012.02039.x

Nelson, C. J., Seiber, J. N., and Brower, L. P. (1981). Seasonal and intraplant variation of cardenolide content in the california milkweed, Asclepiaseriocarpa, and implications for plant defense. J. Chem. Ecol. 7, 981-1010. doi: 10.1007/BF00987622

Oksanen, J., Blanchet, F. G., Kindt, R., Legendre, P., O'Hara, R. B., Simpson, G. L., et al. (2012). Vegan: Community Ecology Package. Available online at: http://cran. r-project.org/web/packages/vegan/ index.html

Öpik, M., Moora, M., Liira, J., and Zobel, M. (2006). Composition of root-colonizing arbuscular mycorrhizal fungal communities in different ecosystems around the globe. J. Ecol. 94, 778-790. doi: 10.1111/j.1365-2745.2006.01136.x

Orme, D., Freckleton, R. P., Thomas, G., Petzoldt, T., Fritz, S., Isaac, N., et al. (2012). Caper: Comparative Analyses of Phylogenetics and
Evolution in R. Available online at: http://cran.r-project.org/web/packa ges/caper/vignettes/caper.pdf

Partida-Martinez, L. P., and Heil, M. (2011). The microbe-free plant: fact or artifact? Front. Plant Sci. 2:100. doi: $10.3389 /$ fpls.2011.00100

Pineda, A., Zheng, S. J., van Loon, J. J. A., Pieterse, C. M. J., and Dicke, M. (2010). Helping plants to deal with insects: the role of beneficial soil-borne microbes. Trends Plant Sci. 15, 507-514. doi: 10.1016/j.tplants.2010.05.007

Rasmann, S., and Agrawal, A. A. (2011a). Evolution of specialization: a phylogenetic study of host range in the red milkweed beetle (Tetraopes tetraophthalmus). Am. Nat. 177, 728-737. doi: 10.1086/ 659948

Rasmann, S., and Agrawal, A. A. (2011b). Latitudinal patterns in plant defense: evolution of cardenolides, their toxicity and induction following herbivory. Ecol. Lett. 14, 476-483. doi: 10.1111/j.14610248.2011.01609.x

Rasmann, S., Agrawal, A. A., Cook, C. S., and Erwin, C. A. (2009). Cardenolides, induced responses in shoots and roots, and interactions between above and belowground herbivores in the milkweeds (Asclepias spp ) Ecology 90, 2393-2404. doi: 10.1890/08-1895.1

Rasmann, S., Erwin, A. C., Halitschke, R., and Agrawal, A. A. (2011). Direct and indirect root defences of milkweed (Asclepias syriaca): trophic cascades, trade-offs and novel methods for studying subterranean herbivory. J. Ecol. 99, 16-25. doi: 10.1111/j.1365-2745.2010.01713.x

Reinhart, K. O., Wilson, G. W. T., and Rinella, M. J. (2012). Predicting plant responses to mycorrhizae: integrating evolutionary history and plant traits. Ecol. Lett. 15, 689-695. doi: 10.1111/j.1461-0248. 2012.01786.x

Rieske, L. K. (2001). Influence of symbiotic fungal colonization on oak seedling growth and suitability for insect herbivory. Environ. Entomol. 30, 849-854. doi: 10.1603/0046225X-30.5.849

Smith, S. E., and Read, D. R., (2008). Mycorrhizal Symbiosis, 3rd Edn. Amsterdam; New York; Boston: Academic Press.

Sternberg, E. D., Lefèvre, T., Li, J., de Castillejo, C. L. F., Li, H., Hunter, M. D., and de Roode, J. C. (2012). Food plant derived disease tolerance and resistance in a natural butterfly-plant-parasite interactions. Evolution 66, 3367-3376. doi: 10.1111/j.1558-5646.2012.01693.x
Stockinger, H., Walker, C., and Schussüler, A. (2009). Glomus intraradices DAOM197198Ć a model fungus in arbuscular mycorrhiza research, is not Glomus intraradices. New Phytol. 183, 1176-1187. doi: 10.1111/j.14698137.2009.02874.x

Strauss, S. Y., and Irwin, R. E. (2004). Ecological and evolutionary consequences of multispecies plantanimal interactions. Annu. Rev. Ecol. Evol. Syst. 35, 435-466. doi: 10.1146/annurev.ecolsys.35.112202. 130215

Tao, L., and Hunter, M. D. (2012). Does anthropogenic nitrogen deposition induce phosphorus limitation in herbivorous insects? Glob. Change Biol. 18, 1843-1853. doi: 10.1111/j.1365-2486.2012.02645.x

Team, R. C. D. (2012). R: A Language and Environment for Statistical Computing. Vienna: R Foundation for Statistical Computing.

Thamer, S., Schadler, M., Bonte, D., and Ballhorn, D. J. (2011). Dual benefit from a belowground symbiosis: nitrogen fixing rhizobia promote growth and defense against a specialist herbivore in a cyanogenic plant. Plant Soil 341, 209-219. doi: 10.1007/s11104-010-0635-4

van Dam, N. M. (2009). Belowground herbivory and plant defenses. Аnпu. Rev. Ecol. Evol. Syst. 40, 373-391. doi: 10.1146/ annurev.ecolsys.110308.120314

van der Meijden, E., Wijn, M., and Verkaar, H. J. (1988). Defense and regrowth, alternative plant strategies in the struggle against herbivores. Oikos 51, 355-363. doi: $10.2307 / 3565318$

Vandenkoornhuyse, P., Husband, R., Daniell, T. J., Watson, I. J., Duck, J. M., Fitter, A. H., and Young, J. P. W. (2002). Arbuscular mycorrhizal community composition associated with two plant species in a grassland ecosystem. Mol. Ecol. 11, 1555-1564. doi: 10.1046/j.1365294X.2002.01538.x

Vannette, R. L., and Hunter, M. D. (2009). Mycorrhizal fungi as mediators of defence against insect pests in agricultural systems. Agric. For. Entomol. 11, 351-358. doi: 10.1111/j.1461-9563.2009.00445.x

Vannette, R. L., and Hunter, M. D. (2011). Plant defence theory re-examined: nonlinear expectations based on the costs and benefits of resource mutualisms. J. Ecol. 99, 66-76. doi: 10.1111/j.1365-2745.2010.01755.x

Vannette, R. L., and Hunter, M. D. (2013). Mycorrhizal abundance affects the expression of plant resistance traits and herbivore performance. J. Ecol. 99, 66-76. doi: 10.1111/1365-2745.12111

Vannette, R. L., and Rasmann, S. (2012). Arbuscular mycorrhizal fungi mediate belowground plant-herbivore interactions: a phylogenetic study. Funct. Ecol. 26, 1033-1042. doi: 10.1111/j.13652435.2012.02046.x

Zalucki, M. P., Brower, L. P., and Alonso, A. (2001a). Detrimental effects of latex and cardiac glycosides on survival and growth of first-instar monarch butterfly larvae Danaus plexippus feeding on the sandhill milkweed Asclepias humistrata. Ecol. Entomol. 26, 212-224. doi: 10.1046/j.1365-2311.2001.00313.x

Zalucki, M. P., Malcolm, S. B., Paine, T. D., Hanlon, C. C., Brower, L. P., and Clarke, A. R. (2001b). It's the first bites that count: survival of first-instar monarchs on milkweeds. Austral Ecol. 26, 547-555. doi: $\quad 10.1046 / j .1442-9993.2001$ 01132.x

Zehnder, C. B., and Hunter, M. D. (2007). Interspecific variation within the genus Asclepias in response to herbivory by a phloem-feeding insect herbivore. J. Chem. Ecol. 33, 2044-2053. doi: 10.1007/s10886-007-9364-4

Conflict of Interest Statement: The authors declare that the research was conducted in the absence of any commercial or financial relationships that could be construed as a potential conflict of interest.

Received: 30 April 2013; accepted: 26 August 2013; published online: 19 September 2013.

Citation: Vannette RL, Hunter MD and Rasmann S (2013) Arbuscular mycorrhizal fungi alter above- and below-ground chemical defense expression differentially among Asclepias species. Front. Plant Sci. 4:361. doi: 10.3389/fpls.2013.00361

This article was submitted to PlantMicrobe Interaction, a section of the journal Frontiers in Plant Science.

Copyright @ $\odot 2013$ Vannette, Hunter and Rasmann. This is an open-access article distributed under the terms of the Creative Commons Attribution License (CC BY). The use, distribution or reproduction in other forums is permitted, provided the original author(s) or licensor are credited and that the original publication in this journal is cited, in accordance with accepted academic practice. No use, distribution or reproduction is permitted which does not comply with these terms. 